\title{
论 文
}

\section{刚柔分级并联驱动宏微复合运动平台设计}

\author{
白有盾, 陈新, 杨志军 ${ }^{*}$ \\ 广东工业大学精密电子制造技术与装备国家重点实验室, 广东省微纳加工技术与装备重点实验室, 广州 510006 \\ *E-mail: yangzj@gdut.edu.cn
}

收稿日期: 2017-11-19; 接受日期: 2018-07-28; 网络版发表日期: 2019-02-28

国家自然科学基金(批准号: 91648108, 51875108, U1601202, 51675106)、广东省自然科学基金(批准号: 2015A030312008, 2015A030308004)、 广东科技计划(编号：2015B010104006，2015B010104008)、国家重点研发计划(编号：2017YFF0105902)、中国博士后科学基金(编号： 2018M643019)和广州科技计划(编号: 201510010281)资助项目

摘要高速精密定位是电子制造的要求, 轴系摩擦是影响精密定位的重要因素. 目前, 微米级以上精度必须通过 消除摩擦影响的方式来实现, 成本很高. 本文将大行程的直线电机平台与无摩擦的柔性铰链导向机构结合, 提出 了一种并联驱动的宏微复合设计新方案, 克服了现有宏微复合平台存在的输出饱和问题. 具体做法是在无铁芯永 磁直线电机模组中, 安装两套驱动和测量反馈系统, 分别用于驱动宏运动平台和与之通过柔性铰链相连的微运动 平台. 宏运动平台通过直线导轨滑块“刚性”机械连接系统实现大行程高速运动，微运动平台通过基于柔性铰链的 “柔性”连接来快速补偿“刚性”宏运动平台的误差, 最终实现大行程高速高精度运动. 本文利用上述刚柔分级运动 方案, 通过基于动力学响应的运动规划方法进一步抑制了高加速运动平台的定位残余振动. 基于柔性铰链的微运 动系统较之宏运动系统具有更快的响应速度和更小的跟踪轨迹误差, 从而实现在高加速运动过程中宏/微驱动力 叠加来实现快速运动, 同时在匀速与低速定位过程中微驱动器产生反方向作用力来快速补偿宏运动平台的运动 误差来实现高定位精度. 实验表明, 本文提出的宏微复合驱动方案, 通过机构的创新, 可有效降低控制的复杂度, 并实现了高精密快速定位, 可以为微光电子制造装备亚微米级高速定位平台设计提供崭新的途径.

关键词 刚柔分级, 并联驱动, 宏微复合, 精密定位

\section{1 引言}

高精度点位运动平台是半导体、液晶面板等电子 制造行业中不可或缺的设备之一，消费电子等行业的 急速发展导致了对各类低成本、高集成度电子产品的 巨大需求，进而对各类精密点位运动平台提出了更高 定位精度与更短定位时间的综合要求. 上述要求意味 着精密点位运动平台必须同时具备频繁启停、高加
速、高定位精度等性能指标. 此外, 电子制造工艺的进 步也导致各类电子芯片晶圆与基板尺寸不断增大，使 得精密点位运动平台也要具有更大的工作行程. 由于 导轨摩擦等非线性因素以及大行程运动平台自身质量 限制, 目前的单级驱动大行程运动平台的定位精度与 控制带宽已经到达一定极限 ${ }^{[1 ~ 3]}$, 很难满足上述更高的 性能要求.

自从 20 世纪 80 年代 $S h a r o n^{[4,5]}$ 在机器人领域提出

引用格式: 白有盾, 陈新, 杨志军. 刚柔分级并联驱动宏微复合运动平台设计. 中国科学: 技术科学, 2019, 49: 669-680 Bai Y D, Chen X, Yang Z J. Design of rigid motion and elastic deformation parallel driven based macro-micro compounded positioning stage (in Chinese). Sci Sin Tech, 2019, 49: 669-680, doi: 10.1360/N092017-00397 
宏微复合驱动的概念后，宏微复合双级驱动就被认为 是一种可以兼顾大行程高速运动与高定位精度的驱动 方式，在硬盘磁头驱动 ${ }^{[6 ~ 10]}$ 以及各类高速精密定位平 台 ${ }^{[1114]}$ 中得到了广泛应用. 宏微复合运动平台主要是 通过在大行程、低带宽、粗精度的宏运动平台上叠加 小行程、高带宽、高精度的微运动平台来实现大行 程、高精度的宏微复合运动. 上述宏/微运动平台之间 的连接形式可以分为机械耦合与机械解耦两种形式. 其中, 前者的宏/微运动平台往往采用压电陶瓷驱动器/ 电磁驱动器等 + 柔性铰链机构等机械方式 ${ }^{[1,15,16]}$ 连接, 后者的宏/微运动平台通常采用电磁驱动器 + 气浮/磁 悬浮等非接触形式 ${ }^{[17 ~ 19]}$ 连接. 由于微驱动器与柔性铰 链机构的工作行程限制, 机械耦合形式的宏微复合运 动平台容易出现微运动平台输出饱和问题, 往往需要 采用宏微切换 ${ }^{[2022]}$ 、主从控制 ${ }^{[23 ~ 25]}$ 、非线性反馈控 制 ${ }^{[26-28]} 、 \mathrm{PQ}$ 补偿器 ${ }^{[29 ~ 32]}$ 等方式来解决上述输出饱和 问题, 增加了控制系统的复杂度, 不利于缩短定位时间 与提高系统可靠性. 机械解耦形式的宏微复合运动平 台中往往采用电磁驱动器作为微驱动器, 其中电磁驱 动器的动子/定子(或定子/动子)分别固定在宏运动平 台与微运动平台上. 由于机械解耦形式的宏微复合运 动平台的机械解耦结构特点，其宏微控制系统可以基 本按照两个独立的单输入单输出(SISO)系统进行设 计，通过给宏/微两套控制系统发送相同的运动规划指 令来控制宏/微运动平台保持一定的相对位移 ${ }^{[17,18]}$. 上 述宏微控制系统避免了微驱动器输出饱和导致的非线 性控制问题，同时也便于配合高速运动规划来进一步 缩短定位时间. 但是上述机械解耦形式宏微复合运动 平台中宏/微平台之间由电磁驱动器提供的相对较小 作用力导致其微运动平台在大加速情况下容易与宏运 动平台发生碰撞，限制了机械解耦形式宏微复合运动 平台的最大加速度极限. 针对上述问题, 日本东京大 学在机械解耦形式宏微复合运动平台的宏/微运动平 台之间设计了碰撞接触装置 ${ }^{[2,33]}$ 和相应的碰撞力控制 模型, 使得宏/微运动平台在加速阶段可以相互碰撞接 触来增大宏/微运动平台的相互作用力, 从而提高微运 动平台的最大加速度极限. 但是上述加速阶段的碰撞 接触工作方式也导致其不适用于频繁加减速的应用场 合. 同时, 碰撞接触力模型的引入也在一定程度上增加 了控制系统的复杂度.

综合分析现有宏微复合运动平台在电子封装领域
常见的频繁高速点位操作存在的不足, 本文设计了一 种针对高速点位运动的宏微复合运动平台, 采用两套 并联布置的绝对位移传感器与直线驱动器来分别构建 独立的宏/微闭环伺服控制系统，避免了微运动平台输 出饱和导致的控制问题. 通过将考虑运动平台动力学 特性影响的时间最优点位运动规划应用到上述宏微双 驱控制系统来进一步缩短宏微复合运动平台的点位运 动定位时间. 本文所设计的宏微复合运动平台中的宏/ 微运动平台之间通过直梁型柔性铰链进行连接，上述 微运动平台可以通过直梁型柔性铰链的连续变形实现 “柔性”的小行程高精度运动，上述宏运动平台通过直 线导轨滑块等“刚性”连接机械系统实现大行程高速度 运动，利用基于直梁型柔性铰链的“柔性”微运动平台 来快速补偿“刚性”宏运动平台的误差, 并最终实现大 行程高速高精度运动. 此外, 利用直梁型柔性铰链在 轴向力作用下的应力刚化效应可以方便地调节“柔性” 微运动平台的固有频率来适应不同应用场合的工作频 率需求. 最后, 本文通过实验测试验证了所设计的新型 宏微运动平台可以有效地抑制运动平台的超调量与定 位过程残余振动，并在保证定位精度的前提下显著缩 短点位运动定位时间. 本文所提出的新型宏微复合运 动平台设计在运动规划、平台结构设计与宏微双驱控 制系统等的一体化综合设计方面做出了尝试，可以为 进一步提高电子封装设备的定位精度与工作效率提供 一种新的设计思路.

\section{2 宏微复合运动平台的技术背景}

如上所述，常见的宏微复合运动平台按照宏/微运 动平台连接形式可以分为机械耦合与机械解耦等形 式, 其结构原理示意图如图1所示. 其中, $F_{1}$ 和 $F_{2}$ 分别为 宏/微运动平台的驱动器作用力, $M$ 和 $m$ 分别为宏/微运 动平台的运动质量， $x_{1}$ 和 $x_{2}$ 分别为宏/微运动平台上的 位移传感器测量值, $k$ 和 $c$ 分别为机械耦合形式宏微复 合运动平台的宏/微运动平台之间连接机构的刚度与 阻尼比. 容易看出, 机械耦合形式的宏微复合运动平台 中的微运动平台驱动器以及连接机构的作用力可以视 为宏运动平台的内力, 这使得机械耦合形式的宏微复 合运动平台只能采用微运动平台闭环系统来补偿宏运 动平台运动误差的双输入单输出方式进行宏微控制系 统设计, 进而导致需要考虑前言所述的微驱动输出饱 

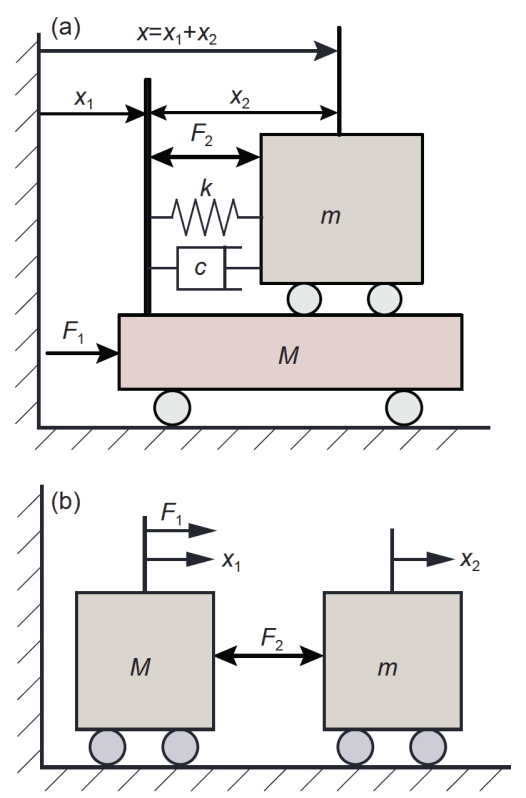

图 1 宏微复合运动平台原理示意图. (a) 机械耦合类型; (b) 机械解耦类型

和问题. 与机械耦合方式不同, 机械解耦形式的宏微复 合运动平台由于采用气浮/磁悬浮支承，其机械连接形 式得以极大简化，对应的耦合连接作用力由微驱动器 作用力 $F_{2}$ 提供. 上述 $F_{2}$ 本质上也属于宏运动平台的内 力，但通过简单的作用力变换可以获得其宏/微运动平 台的等效驱动力, 进而将机械解耦形式宏微复合运动 平台的双输入单输出(DISO)双驱控制系统设计等效分 解为两套相对独立的单输入单输出(SISO)控制系统设 计. 通过向上述两套SISO控制系统发送相同的运动规 划指令, 即可等效实现宏微DISO控制.

\section{3 新型宏微复合运动平台设计}

\section{1 结构设计方案}

由于运动规划对于缩短点位运动的定位时间与抑 制振动影响较大 ${ }^{[34]}$, 并且基于上节的讨论分析，显然 采用机械解耦形式宏微运动平台所用的双驱控制系统 模型有利于各种高速点位运动规划方法的应用. 但是 如上所述, 图1(b)所示的机械解耦形式宏微复合运动 平台由于宏/微作用力 $F_{2}$ 相对较小而不适合于大加速 场合, 而日本东京大学提出的改进平台 ${ }^{[2]}$ 又不适合频 繁加减速场合. 同时考虑到机械耦合连接方式对于提 高宏/微之间连接作用力的优点，本文提出了图2所示
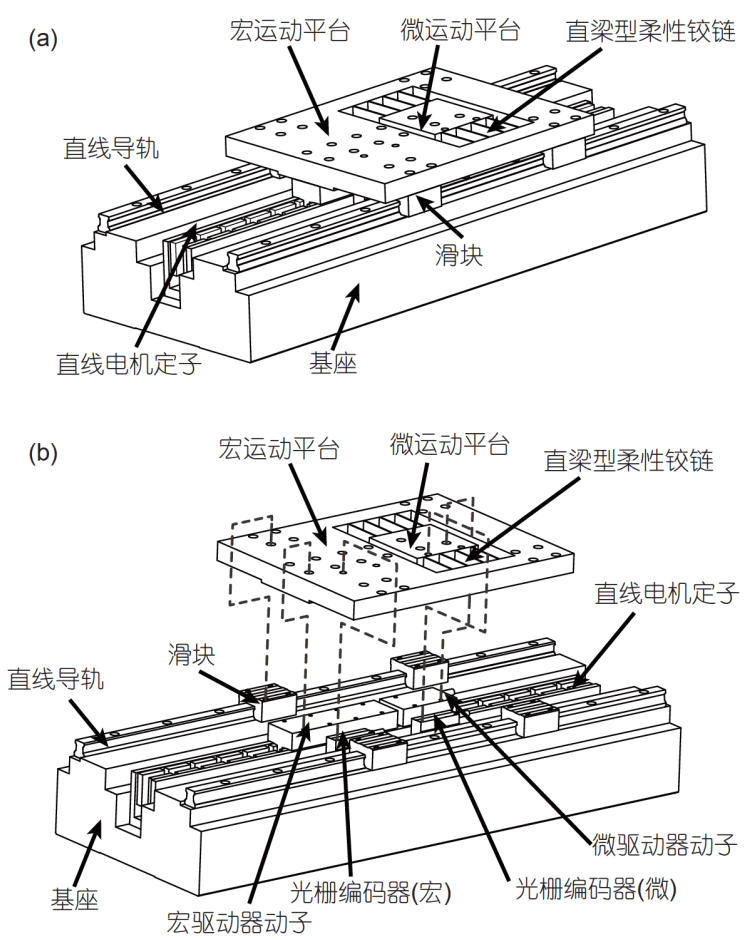

图 2 新型宏微复合运动平台结构示意图

的新型宏微复合运动平台设计.

如图2所示, 本文所设计的新型宏微复合运动平台 采用了共用直线电机定子的并联驱动形式的设计方 案. 其中, 宏运动平台通过直线导轨滑块系统与基座之 间刚性连接, 微运动平台之间通过直梁型柔性铰链与 宏运动平台之间柔性连接, 宏/微运动平台包含有相互 独立的光栅位移编码器与驱动器动子(图2(b)). 上述 宏/微运动平台的电磁驱动力均直接作用在与基座刚 性连接的直线电机定子上, 同时两套独立的光棶位置 传感器也便于双反馈闭环伺服系统的构建. 图2中的 宏微复合运动平台与现有宏微复合运动平台的主要区 别之处为: (1) 宏/微运动平台内驱动器的驱动力均直 接作用在基座上，便于实现宏/微独立伺服控制; (2) 宏/ 微运动平台之间采用无摩擦的直梁型柔性铰链作为连 接机构, 便于微运动平台补偿宏运动平台的定位误差; (3) 直梁型柔性铰链可以通过调整其轴向力的方式来 动态调整工作刚度, 并进而更改微运动平台的工作频 率, 有利于宏微复合运动平台适应不同应用场合的频 率需求.

图2所示的新型宏微复合运动平台的工作原理可 以表示为双质量弹簧振动系统(图3). 其中, $F_{1}$ 和 $F_{2}$ 分别 


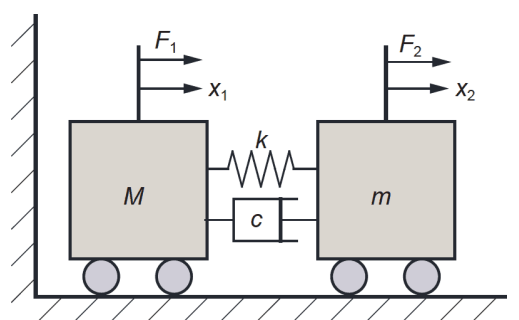

图 3 新型宏微复合运动平台的等效动力学系统示意图

为宏/微运动平台的驱动器作用力, $M$ 和 $m$ 分别为宏/微 运动平台的运动质量, $x_{1}$ 和 $x_{2}$ 分别为宏/微运动平台上 的位移传感器测量值, $k$ 和 $c$ 分别为直梁型柔性铰链机 构的刚度与阻尼比.

\section{2 微运动平台设计}

由于宏/微运动平台之间的质量差异很大 $(M \gg m)$, 且微运动平台的本体刚度要远大于柔性铰 链的工作刚度, 微运动平台的动力学特性主要取决于 柔性铰链. 因此, 可以将图2中所示的直梁型柔性铰链 视为微运动平台的组成部分.

图2所示的单片直梁型柔性铰链可以表示为图4所 示的一端固定一端导向梁.

根据文献[35]中提出的计算方法, 图4所示的单片 直梁型柔性铰链的工作刚度与承载刚度分别为

$$
\begin{aligned}
& k_{\mathrm{d}}=E w\left(\frac{t}{L}\right)^{3}, \\
& k_{\mathrm{p}}=E t\left(\frac{w}{L}\right)^{3},
\end{aligned}
$$

式中, $E, w, t$ 和 $L$ 分别为图2中单片直梁型柔性铰链的 杨氏模量、宽度、厚度与长度.

由于应力刚化效应, 图2中单片直梁型柔性铰链在 承受大轴向力时，其工作刚度会显著增大. 利用文献 [35]中的轴向张紧力刚度计算方式，式(1)所示工作刚 度公式可以修正为

$k_{\mathrm{d}}^{\prime}=E w\left(\frac{t}{L}\right)^{3}+\frac{13 \pi^{2}}{140}\left(\frac{N}{L}\right)$,

式中, $N$ 为单片直梁型柔性铰链的轴向力.

图2中微运动平台与直梁型柔性铰链组构成的柔 性动力学系统的结构质量由微运动平台质量 $m$ 与直梁 型柔性铰链的等效质量组成. 其中单片直梁型柔性铰

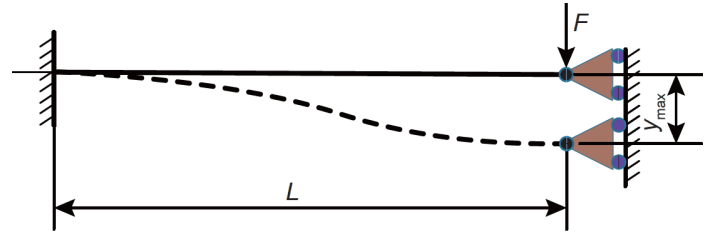

图 4 (网络版彩图)单片直梁型柔性铰链变形示意图

链的等效质量利用等效能量方法 ${ }^{[35]}$, 可以表示为

$m_{\mathrm{e}}=\frac{13}{35} m_{\text {柔性铰链 }}=\frac{13}{35} \rho w t L$.

利用式(3)和(4), 可以得到中直梁型柔性铰链组与 微运动平台的工作频率:

$$
\begin{aligned}
& f_{\text {微 }}=\frac{1}{2 \pi} \sqrt{\frac{k_{\text {总 }}}{m_{\text {总 }}}}=\frac{1}{2 \pi} \sqrt{\frac{n k_{\mathrm{d}}^{\prime}}{m+n m_{\text {柔性铰链 }}}} \\
& =\frac{1}{2 \pi} \sqrt{\frac{n E w\left(\frac{t}{L}\right)^{3}+\frac{13 n \pi^{2}}{140}\left(\frac{N}{L}\right)}{m+(13 / 35) n \rho w t L}},
\end{aligned}
$$

式中, $n$ 为直梁型柔性铰链的片数.

由式(3)与(5), 图2所示的直梁型柔性铰链与微运 动平台组成柔性系统的工作刚度与工作频率均受直梁 型柔性铰链的轴向力影响. 上述直梁型柔性铰链的轴 向力包括: 主动施加轴向力 $N_{\text {主动 }}$ 与直梁型柔性铰链在

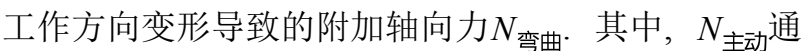
常为恒定值, 用于调节直梁型柔性铰链的刚度来改变 微运动平台的固有频率来适应不同工作频率的应用场 合需求. $N_{\text {弯曲随直梁型柔性铰链的变形量增加而非线 }}$ 性增大，导致非线性刚度问题. 为了避免由于 $N_{\text {弯曲所 }}$ 导致的非线性刚度问题，本文根据图4中所示驱动力 $F$ 幅值与最大工作行程 $y_{\text {max }}$ 等要求来设计适当的直梁型 柔性铰链工作刚度, 使得 $y_{\text {max }}<L L$, 从而保证直梁型柔 性铰链在完整工作行程中始终处于 $N_{\text {弯曲 }} \approx 0$ 的小变形 工况.

\section{3 新型宏微复合运动平台设计配置}

根据上述结构设计方案与微运动平台的设计方 法, 本文设计并加工制造了新型宏微复合运动平台, 如 图5所示.

如图5所示, 本文所设计的新型宏微复合运动平台 


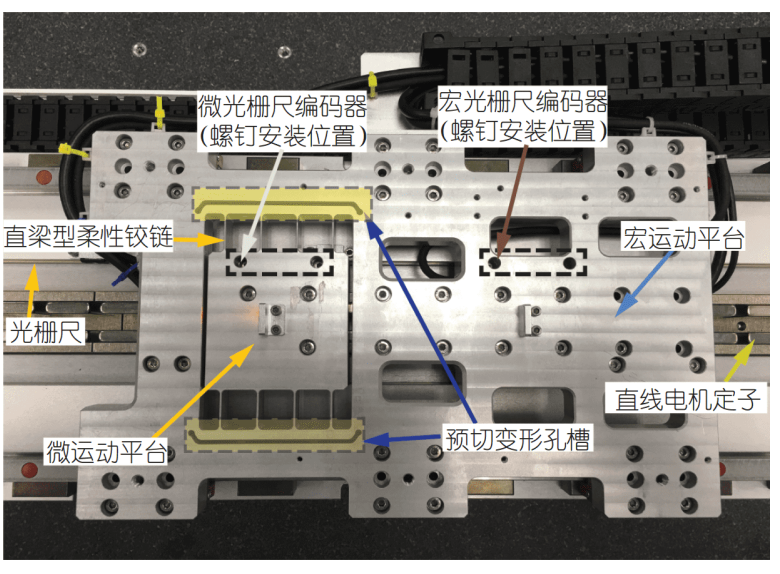

图 5 (网络版彩图)加工制造的新型宏微复合运动平台

采用了无铁芯永磁直线电机(Akribis, AUM3)作为宏/ 微运动平台的驱动器. 其中, 宏/微运动平台分别与相 应的直线电机动子连接，上述直线电机动子共用相同 的直线电机定子. 宏/微运动平台设置有独立的光栅位 移传感器(Renishaw, RGH22). 宏运动平台与图5所示 直梁型柔性铰链连接处设置有预切变形孔槽, 便于通 过螺栓组或压电陶瓷堆栈等来主动调节上述直梁型柔

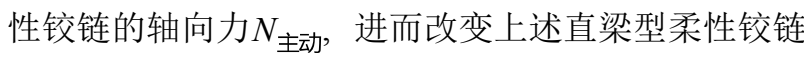
的刚度、频率特性以适应不同应用场合.

根据宏运动平台的直线电机与伺服驱动器的精度 指标, 选定 $\pm 20 \mu \mathrm{m}$ 作为微运动平台的工作行程. 此外, 为了保证微运动平台的快速响应特性，本文在微运动 平台驱动器满足工作行程需求的前提下对直梁型柔性 铰链采用了大刚度设计。

图5所示的新型宏微复合运动平台中的宏/微运动 平台本体均采用7075铝合金加工制造，相应的结构尺 寸及电机参数汇总列在表 1 中.

\section{4 控制系统设计与运动规划}

\section{1 宏微双驱控制系统设计}

由于导轨摩擦、平台结构质量等方面存在的差 异，通常而言宏运动平台的响应速度低于微运动平台, 导致其伺服控制系统的轨迹跟踪误差等要大于微运动 平台. 因此，如果给宏/微运动平台的伺服控制系统发 送相同的运动规划指令, 宏/微运动平台在运动过程中 的相对位置会发生变化，从而导致图5所示宏/微运动 平台之间的直梁型柔性铰链产生变形连接作用力. 如
表 1 宏微复合运动平台的结构尺寸与电机参数

\begin{tabular}{|c|c|c|}
\hline \multicolumn{2}{|l|}{ 参数 } & 数值 \\
\hline \multicolumn{2}{|c|}{$\begin{array}{l}\text { 微运动平台质量 } \\
\text { (含驱动器/传感器) }\end{array}$} & $0.415 \mathrm{~kg}$ \\
\hline \multicolumn{2}{|c|}{$\begin{array}{c}\text { 宏运动平台质量 } \\
\text { (含滑块/驱动器/传感器) }\end{array}$} & $4.190 \mathrm{~kg}$ \\
\hline \multicolumn{2}{|c|}{ 微驱动器峰值推力 } & $144 \mathrm{~N}$ \\
\hline \multicolumn{2}{|c|}{ 宏驱动器峰值推力 } & $433 \mathrm{~N}$ \\
\hline \multirow{3}{*}{ 单片柔性铰链尺寸 } & 宽度 & $18.0 \mathrm{~mm}$ \\
\hline & 长度 & $20.0 \mathrm{~mm}$ \\
\hline & 厚度 & $1.03 \mathrm{~mm}$ \\
\hline
\end{tabular}

图2所示，本文所提出的新型宏微复合运动平台的宏/ 微运动平台的驱动力均直接作用在基座上，且两者均 有相互独立的光栅位置编码器. 因此, 在相同的运动 规划参考指令作用下, 轨迹跟踪误差较小的微运动平 台可以利用直梁型柔性铰链连接机构来动态地修正补 偿宏运动平台的位置误差. 基于上述分析讨论, 针对图 5 所示的宏微复合运动平台的结构特点, 本文提出了图 6所示的宏微双驱控制系统设计.

由于图5所示的宏微复合运动平台中的直梁型柔 性铰链采用了大刚度设计, 微运动平台的工作行程 $( \pm 20 \mu \mathrm{m})$ 远远小于表 1 中所列的直梁型柔性铰链长度 $(20 \mathrm{~mm})$, 上述工况下所述直梁型柔性铰链均处于小变 形状态, 对应的柔性铰链刚度可以视为线性刚度.

图6所示的宏微双驱控制系统实质上是通过给宏/ 微运动平台各自的闭环伺服系统发送相同的运动参考 指令, 利用高带宽、响应速度快的微运动平台来动态 地修正宏运动平台的误差. 上述宏微双驱控制系统与 图2所示的宏/微运动平台并联驱动的结构特点使得微 运动平台可以利用直接作用在与基座刚性连接的直线 电机定子上的电磁力来最大程度抑制高速点位运动历 程中的宏运动平台超调量, 更有效地抑制残余振动.

\section{2 高速点位运动规划设计}

图6所示的宏微双驱控制系统保证了微运动平台 可以有效地补偿宏运动平台的运动误差来提高定位精 度. 为了进一步缩短点位运动定位时间, 还需要设计相 应的时间最优运动规划.

在工程实践中, $\mathrm{S}$ 曲线运动规划被证明是一种可以 缩短定位时间并避免激发过大残余振动的有效方 


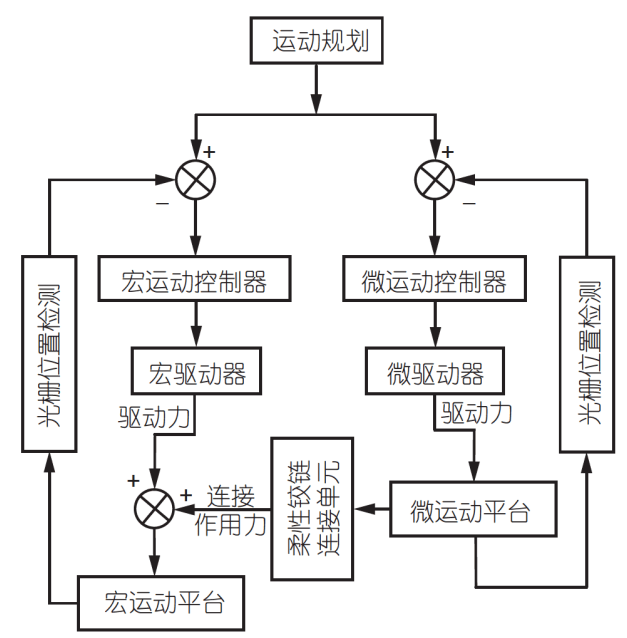

图 6 宏微双驱控制系统示意图

法 $^{[66-38]}$. 此外, 有别于现有的对称 $\mathrm{S}$ 曲线运动规划, 一 种可以实现高加速低减速点位运动的非对称 $\mathrm{S}$ 曲线运 动规划 ${ }^{[39 \sim 42]}$ 被认为可以更好地抑制柔性运动平台的 残余振动并缩短定位时间. 为了进一步缩短高精度点 位运动的定位时间，本文重新定义了一种具有更多独 立设计变量的非对称 $\mathrm{S}$ 曲线, 并采用文献[43]中提出的 考虑系统动力学特性的运动规划优化模型对上述重新 定义的非对称 $\mathrm{S}$ 曲线的参数进行了优化.

本文重新定义的非对称 $\mathrm{S}$ 曲线运动规划如图7所 示, 其与现有非对称 $\mathrm{S}$ 曲线运动规划的主要区别在于将 所有的急动度都设置为独立变量.

图7所示的非对称 $\mathrm{S}$ 曲线运动规划的急动度定义 如下:

$\operatorname{Jerk}(t)=\left\{\begin{array}{l}J_{1},\left(0<t \leq t_{1}\right) \\ -J_{2},\left(t_{2}<t \leq t_{3}\right) ; \\ -J_{3},\left(t_{4}<t \leq t_{5}\right) \\ J_{4},\left(t_{6}<t \leq t_{7}\right) \\ 0, \quad(\text { otherwise })\end{array}\right.$

式中, $J_{i}$ 为急动度幅值, $t_{i}$ 为运动规划曲线的时间参数. 定义 $T_{i}=t_{i}-t_{i-1}(i=1,2 \ldots, 7)$.

式(6)的包含急动度与时间参数在内的 11 个参数 需要满足约束条件: (1) 匀速段中的加速度为 $0 ;$; (2) 运 动终止时刻的加速度为 0 ; (3) 运动终止时刻的速度为 0 ; (4) 运动终止时刻的位移为 $Q$. 上述约束条件可以表 示为

$$
\left\{\begin{array}{l}
\left\{\begin{array}{l}
J_{1} T_{1}-J_{2} T_{3}=0, J_{3} T_{5}-J_{4} T_{7}=0, \\
J_{1} T_{1}\left(T_{1}+2 T_{2}+T_{3}\right)-J_{3} T_{5}\left(T_{5}+2 T_{6}+T_{7}\right)=0 ;
\end{array}\right. \\
\left(\begin{array}{l}
\frac{1}{4} J_{1} T_{1}\left(T_{1}+2 T_{2}+T_{3}\right)\left(2 T_{4}+2 T_{5}+2 T_{6}+T_{3}+T_{7}\right) \\
+\frac{1}{24} J_{1} T_{1}\left(T_{1}^{2}-T_{3}^{2}\right)-\frac{1}{24} J_{3} T_{5}\left(T_{5}^{2}-T_{7}^{2}\right)
\end{array}\right)=Q .
\end{array}\right.
$$

求解式(7), 可得

$J_{i}=\frac{24 Q}{L} \times \frac{\left|G_{i}\right|}{T_{2 i-1}},(i=1,2,3,4)$,

式中,

$G_{1}=-G_{2}=T_{5}+2 T_{6}+T_{7}$,

$G_{3}=-G_{4}=-\left(T_{1}+2 T_{2}+T_{3}\right)$,

$L=3\left(T_{1}+2 T_{2}+T_{3}\right)\left(T_{5}+2 T_{6}+T_{7}\right)\left(\begin{array}{l}T_{1}+2 T_{2}+3 T_{3}+4 T_{4} \\ +3 T_{5}+2 T_{6}+T_{7}\end{array}\right)$

$+\left(T_{5}+2 T_{6}+T_{7}\right)\left(T_{1}^{2}-T_{3}{ }^{2}\right)-\left(T_{1}+2 T_{2}+T_{3}\right)\left(T_{5}{ }^{2}-T_{7}{ }^{2}\right)$.

利用式(6)与(8), 图7中的加速度、速度、位移等 规划曲线可以定义为

$$
\begin{aligned}
& \operatorname{Acc}(t)=\frac{24 Q}{L} \sum_{i=1}^{4} \frac{G_{i}}{T_{2 i-1}}\left(S_{2 i-2}-S_{2 i-1}\right), \\
& \operatorname{Vel}(t)=\frac{12 Q}{L} \sum_{i=1}^{4} \frac{G_{i}}{T_{2 i-1}}\left(S_{2 i-2}^{2}-S_{2 i-1}^{2}\right), \\
& \operatorname{Dis}(t)=\frac{4 Q}{L} \sum_{i=1}^{4} \frac{G_{i}}{T_{2 i-1}}\left(S_{2 i-2}^{3}-S_{2 i-1}^{3}\right),
\end{aligned}
$$

式中,

$S_{i}=\left(t-t_{i}\right) \times$ Heaviside $\left(t-t_{i}\right)$,

$(i=0,1,2, . ., 7), t_{0}=0$.

根据图7和式(6), 可以得到非对称 $\mathrm{S}$ 曲线运动规划 物理约束条件:

$\left\{\begin{array}{l}0<J_{1}, J_{2}, J_{3}, J_{4} \leq J_{\mathrm{m}}, \\ A_{1}=J_{1} T_{1} \leq A_{\mathrm{m}}, A_{2}=J_{3} T_{5} \leq A_{\mathrm{m}}, \\ V_{0}=J_{1} T_{1}\left(T_{1}+2 T_{2}+T_{3}\right) / 2 \leq V_{\mathrm{m}},\end{array}\right.$

式中, $J_{\mathrm{m}}, A_{\mathrm{m}}$ 和 $V_{\mathrm{m}}$ 分别为许用最大急动度、许用最大 加速度、许用最大速度.

根据图6所示宏微双驱控制系统的工作方式，图5 所示的宏微复合运动平台的高速点位运动规划需要满 足如下定位误差约束条件一一宏运动平台在运动规划 指令作用下所导致微运动平台的末段定位过程残余振 动振幅不超过微运动平台的误差补偿能力. 

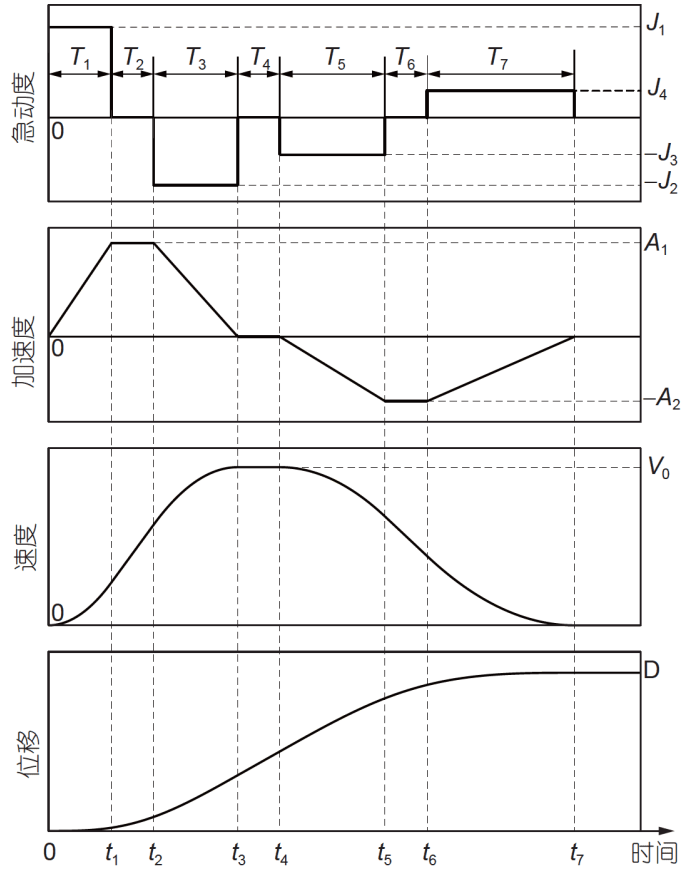

图 7 非对称 $\mathrm{S}$ 运动规划曲线示意图

令微运动平台的驱动力 $F_{2}=0$, 则图3所示新型宏 微复合运动平台对应的双质量运动系统的动力学方 程为

$m \ddot{x}_{2}+c\left(\dot{x}_{2}-\dot{x}_{1}\right)+k\left(x_{2}-x_{1}\right)=0$.

令 $x_{3}=x_{2}-x_{1}$, 则 $x_{3}$ 与 $x_{1}$ 之间的传递函数为

$\frac{X_{3}(s)}{X_{1}(s)}=\frac{-s^{2}}{s^{2}+2 s \omega_{n} \xi+\omega_{n}^{2}}$,

式中, $X_{1}(s)$ 与 $X_{3}(s)$ 分别为宏运动平台绝对位移与微运 动平台相对于宏运动平台的弹性振动位移的Laplace 频域表达式, $\omega_{n}$ 与 $\xi$ 分别为双质量运动系统的固有频率 $\left(\omega_{n}=\sqrt{k / m}\right)$ 与阻尼比 $(\xi=c / 2 \sqrt{m k})$.

设定式(9)对应的加速度运动规划为双质量运动 系统中宏运动平台的系统输入. 其对应的Laplace表达 式为

$s^{2} X_{1}(s)=\frac{24 Q}{L} \times \frac{1}{s^{2}} \sum_{i=1}^{4} \frac{G_{i}}{T_{2 i-1}}\left(\begin{array}{c}\exp \left(-s t_{2 i-2}\right) \\ -\exp \left(-s t_{2 i-1}\right)\end{array}\right)$.

将式(15)代入(14), 可以方便地利用Laplace逆变换 得到微运动平台弹性振动 $x_{3}$ 的位移与速度表达式. 利 用文献[43]中提出的误差定义方法将本文高速运动规
划优化模型中的定位误差约束条件表示为

$\sqrt{d^{2}(t)+\left(v(t) / \omega_{n}\right)^{2}} \leq \varepsilon$,

式中, $d(t)$ 和 $v(t)$ 分别为微运动平台弹性振动 $x_{3}$ 的位移 与速度, $\varepsilon$ 为根据微运动平台的工作行程确定的 $x_{3}$ 许用 最大误差.

基于式(12)和(16)定义的约束条件，可以将时间最 优非对称 $\mathrm{S}$ 曲线运动规划参数优化模型定义为

Find $T_{i}(i=1,2, . ., 7)$

to $\min \sum_{i=1}^{7} T_{i}$

s.t. $\left\{\begin{array}{l}0<J_{1}, J_{2}, J_{3}, J_{4} \leq J_{\mathrm{m}}, \\ J_{1} T_{1} \leq A_{m}, J_{3} T_{5} \leq A_{\mathrm{m}}, \\ J_{1} T_{1}\left(T_{1}+2 T_{2}+T_{3}\right) / 2 \leq V_{\mathrm{m}}, \\ \sqrt{d^{2}(t)+\left(v(t) / \omega_{n}\right)^{2}} \leq \varepsilon .\end{array}\right.$

利用式(17)所述优化模型可以得到匹配实际柔性 运动系统动力学特性且满足微运动平台工作行程需求 的时间最优非对称 $\mathrm{S}$ 曲线运动规划参数值.

\section{5 实验验证}

为了验证本文所设计的新型宏微复合运动平台在 高速点位运动中的工作性能指, 我们搭建了图8所示的 实验测试系统.

如图8所示, 本文设计的新型宏微复合运动平台的 宏/微运动平台通过共用一套定子的两个无铁芯永磁 直线电机(Akribis Inc., 新加坡)动子的电磁力驱动. 上 述永磁直线电机由带光栅尺(Renishaw Inc., 英国)反馈 的伺服控制控制器驱动. 一个 8 轴运动控制卡(固高科 技)被用于生成相应的非对称 $\mathrm{S}$ 曲线运动规划指令. 上 述运动规划指令通过运动控制卡接线板直接发送给相 应的宏/微伺服驱动器, 并驱动宏微复合运动平台. 采 用激光多普勒测振仪(PSV-400-M2, Polytec Inc., 德国) 测试宏/微运动平台的速度响应与动力学参数. 宏/微运 动平台通过各自的光栅尺反馈信号获取.

利用力锤敲击法并配合激光多普勒测振仪, 可以 获得直梁型柔性铰链组与微运动平台构成的柔性运动 系统的固有频率和时域振动衰减曲线(图9). 利用所获 得的时域振动衰减驱动与固有频率值可以计算出上述 柔性运动系统的阻尼比. 


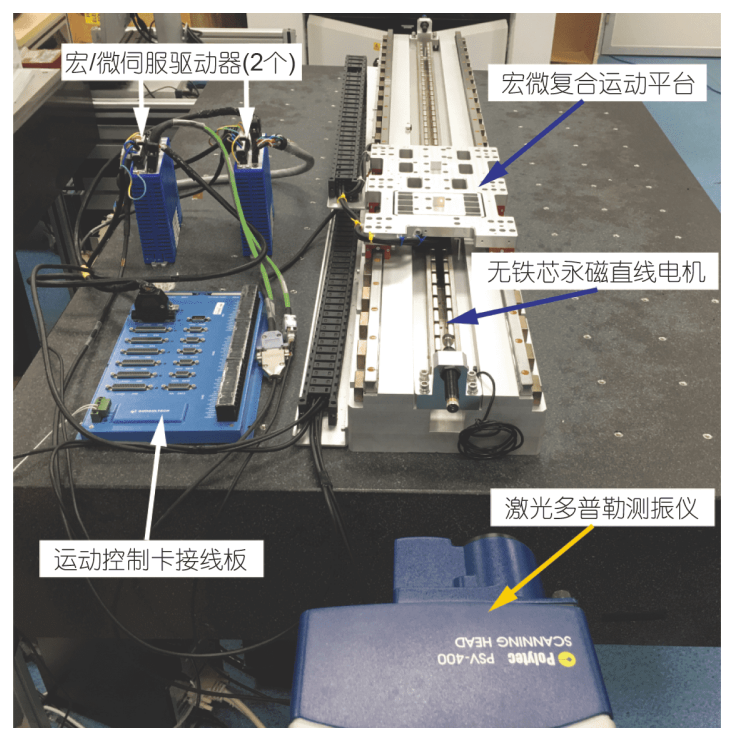

图 8 (网络版彩图)实验测试系统
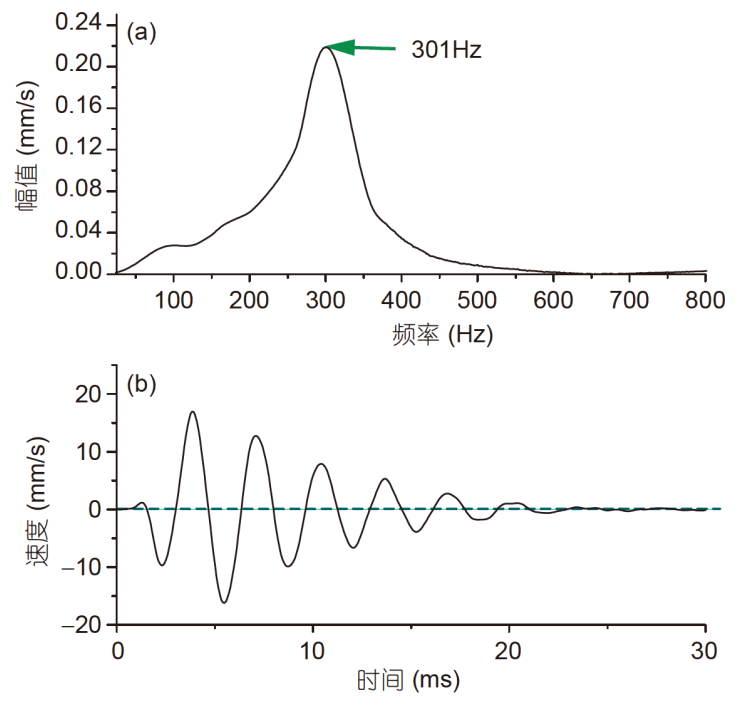

图 9 (网络版彩图)柔性系统的测试频谱图(a)与时域振荡 衰减曲线(b)

根据所采用的永磁直线电机与光栅尺等的性能来 确定后续实验中所用的实验测试参数. 上述实验测试
参数与微运动平台 (含直梁型柔性铰链) 的系统动力学 参数汇总列在表 2 中.

将表 2 中系统动力学参数代入上节提出的运动规 划模型, 可以得到行程 $50 \mathrm{~mm}$ 对应的运动规划参数.

为了验证本文所提的运动规划优化模型在抑制柔 性系统残余振动方面的有效性, 本文将表3中的两组运 动规划参数分别输入宏运动控制器, 并测量微驱动器 不工作(驱动力为零)条件下的微运动平台的速度响应. 所得的速度响应对比曲线绘制在图10中.

根据图10所列的速度响应对比曲线容易看出: 由 于大加速度 $(10 \mathrm{~g})$ 导致的惯性力与微运动平台的柔性 铰链连接方式，文献[36]和本文所述方法所得运动规 划对应的微运动平台的速度响应均存在较大的超调 量. 但是本文所述方法所得运动规划对应的微运动平 台在定位过程的残余振动振幅要明显小于文献[36], 约为后者的 $50 \%$. 这表明本文所提出的运动规划优化 方法有助于缩短点位运动时间, 并便于后续微运动平 台快速补偿上述残余振动误差.

为了验证本文提出的宏微双驱控制系统的有效 性, 我们对相同运动规划参数(表3中(2)组运动规划参 数) 作用下的仅宏驱动器工作与宏/微驱动器协同工作 模式下的微运动平台的速度响应进行了对比测试. 相 关测试结果如图11所示. 可知, 仅宏驱动器工作模式 对应的微运动平台在加速与减速阶段均要滞后于宏/ 微驱动器协同工作模式, 这表明在点位运动过程的加 减速等要求大驱动力的工作阶段, 本文所提的宏微双 驱控制系统可以实现宏/微驱动力叠加来改善宏微复

表 2 实验测试系统参数

\begin{tabular}{cc}
\hline 参数 & 数值 \\
\hline 柔性系统固有频率 & $301 \mathrm{~Hz}$ \\
柔性系统阻尼比 & 0.078 \\
许用最大急动度 & $10000 \mathrm{~m} / \mathrm{s}^{3}$ \\
许用最大加速度 & $100 \mathrm{~m} / \mathrm{s}^{2} \approx 10 g$ \\
许用最大速度 & $1 \mathrm{~m} / \mathrm{s}$ \\
\hline
\end{tabular}

表 3 行程 $50 \mathrm{~mm}$ 对应的点位运动规划参数 ${ }^{\mathrm{a}}$ (单位: $\mathrm{ms}$ )

\begin{tabular}{ccccccccc}
\hline & $T_{1}$ & $T_{2}$ & $T_{3}$ & $T_{4}$ & $T_{5}$ & $T_{6}$ & $T_{7}$ & $T_{\text {sum }}$ \\
\hline (1) & 2.0 & 8.0 & 2.0 & 38.0 & 2.0 & 8.0 & 2.0 & 62.0 \\
$(2)$ & 2.0 & 8.0 & 2.0 & 35.4 & 3.0 & 10.9 & 3.3 & 64.6 \\
\hline
\end{tabular}

a) (1)采用文献[36]中的对称 $\mathrm{S}$ 曲线运动规划模型, (2)采用本文提出的时间最优非对称 $\mathrm{S}$ 曲线运动规划模型. 


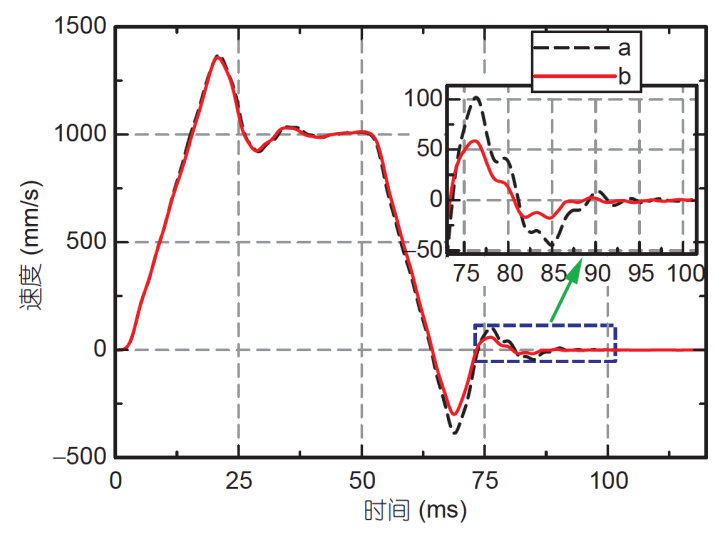

图 10 (网络版彩图)不同运动规划参数作用下的微运动平 台速度响应. (a) 采用文献[36]所述方法; (b) 采用本文所提 方法

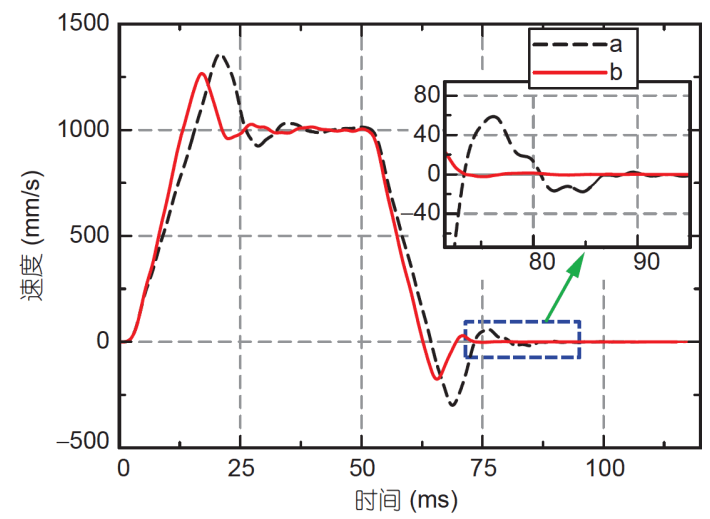

图 11 (网络版彩图)不同宏/微驱动器工作模式对应的微运 动平台速度响应. (a) 仅宏驱动器工作模式; (b) 宏/微驱动器 协同复合工作模式

合运动平台的快速响应特性. 同时, 在匀速及最终的定 位过程中, 宏/微驱动器协同工作模式对应的超调量以 及残余振动振幅要小于仅宏驱动器工作模式，这表明 在匀速等平稳运动阶段, 微驱动器可以有效地补偿宏 运动平台的运动误差. 由速度响应局部放大图(图11) 容易看出：在定位阶段，宏/微协同工作模式对应微运 动平台几乎是“瞬间”满足定位精度要求，而仅宏驱动 器工作模式对应的微运动平台则必须依赖于系统阻尼 与较长的振动衰减时间才能满足定位精度要求. 上述 不同工作模式对应的微运动平台速度响应对比测试结 果验证了本文所提出的宏微双驱控制系统可以有效地 改善宏微复合运动平台的快速响应特性，并快速抑制 定位过程中的残余振动.
最后，本文通过将微运动平台与宏运动平台刚性 锁定得到一个驱动力为宏/微驱动器驱动力之和的等 效单驱运动平台, 并测试了表3中(2)组运动规划参数作 用下的上述单驱运动平台的速度响应. 上述单驱运动 平台速度响应与图11中的宏/微协同工作模式对应的 宏微双驱运动平台中微运动平台速度响应共同绘制在 图12中.

根据图12可以看出, 在加减速等阶段, 单驱运动平 台与宏微双级驱动运动平台中微运动平台的速度响应 曲线基本重合，表明宏微双级驱动平台在加减速阶段 可以基本实现与大驱动力单级运动平台类似的高加速 能力. 在匀速与末段定位阶段, 可以看出宏微双级驱动 运动平台的速度超调量要小于单驱运动平台. 尤其是 在接近 0 速度的定位过程中, 宏微双级驱动运动平台 可以快速抑制残余振动, 而单驱运动平台则需要一定 的振动衰减时间才能满足定位精度要求. 选取 $\pm 2 \mu \mathrm{m}$ 作为最终许用位移定位误差 (对应速度误差约为 $\pm 3.8 \mathrm{~mm} / \mathrm{s}$ ), 则单驱运动平台与宏微双驱运动平台的 定位时间分别为 85 和 $73 \mathrm{~ms},\left(t_{\mathrm{D}}-t_{\mathrm{S}}\right) / t_{\mathrm{S}}=-14.1 \%$. 此对 比结果验证了本文所设计的新型宏微复合运动平台在 缩短点位运动时间方面的有效性.

\section{6 结论}

宏微复合双级驱动是一种实现高速高精密的有效 方式, 被广泛应用在半导体制造等领域. 其中, 宏微复 合双级驱动运动平台的设计重点主要为宏/微连接方 式与宏微双驱控制系统等方面. 本文通过分析现有机

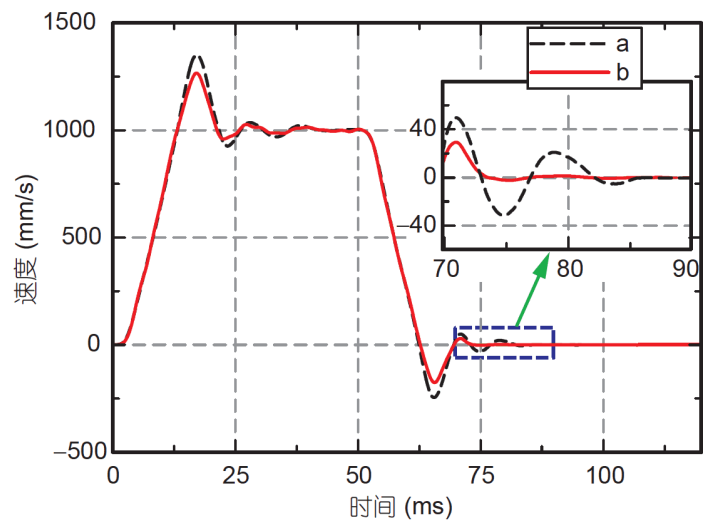

图 12 (网络版彩图)单级驱动平台与宏微双级驱动平台的 微运动平台速度响应. (a) 单级驱动运动平台; (b) 宏微双级 驱动运动平台 
械耦合与机械解耦连接形式的宏微复合运动平台在结 构形式与宏微双驱控制系统设计方面的优缺点, 提出 了一种刚柔分级并联驱动形式的宏微复合运动平台结 构与宏微复合控制系统设计. 通过共用直线电机定子 的宏/微驱动器并联驱动布置方式, 避免了微驱动器输 出饱和问题，简化了宏微复合双驱控制系统设计. 最 后，提出了一种针对宏微复合运动平台动力学特性的
高速点位运动规划优化模型，从运动能量输入角度来 避免激发过大的定位残余振动，进一步缩短宏微复合 运动平台的定位时间. 实验测试结果验证了所提出的 新型宏微复合运动平台可以有效地缩短高精密点位运 动的定位时间. 本文所提出的宏微复合运动平台的结 构与控制系统设计方案为微光电子制造装备亚微米级 高速定位平台设计提供了崭新的途径.

致谢硕士研究生张炫山、何海超、何耀滨等为本文的实验测试工作提供了帮助, 一并致谢.

\section{参考文献}

1 Zhu H, Pang C K, Teo T J. Integrated servo-mechanical design of a fine stage for a coarse/fine dual-stage positioning system. IEEE/ASME Trans Mechatron, 2016, 21: 329-338

2 Yazaki Y, Fujimoto H, Hori Y, et al. Method of shortening settling time using final state control for high-precision stage with decouplable structure of fine and coarse parts. Electr Eng Jpn, 2016, 195: 39-49

3 Zhu H, Pang C K, Teo T J. A flexure-based parallel actuation dual-stage system for large-stroke nanopositioning. IEEE Trans Ind Electron, 2017, 64: 5553-5563

4 Sharon A, Hardt D. Enhancement of robot accuracy using endpoint feedback and a macro-micro manipulator system. In: 1984 American Control Conference. San Diego: IEEE, 1984. 1836-1845

5 Sharon A. The macro/micro manipulator: An improved architecture for robot control. Dissertation of Masteral Degree. Massachusetts: Massachusetts Institute of Technology, 1988

6 Yang J P, Lau G K, Tan C P, et al. Improved design of polymeric composite electrothermal micro-actuator for high track density hard disk drives. Microsyst Technol, 2013, 19: 1697-1704

7 Huang D, Venkataramanan V, Xu J X, et al. Contact-induced vibration in dual-stage hard disk drive servo systems and its compensator design. IEEE Trans Ind Electron, 2014, 61: 4052-4060

8 Sonkham S, Pinsopon U, Chatlatanagulchai W. A model-reference sliding mode for dual-stage actuator servo control in HDD. In: 2014 11th International Conference on Electrical Engineering/Electronics, Computer, Telecommunications and Information Technology. Nakhon Ratchasima: IEEE, 2014. 1-6

9 Rahman M A, Al Mamun A, Yao K. Rate dependent direct inverse hysteresis compensation of piezoelectric micro-actuator used in dual-stage hard disk drive head positioning system. Rev Sci Instrum, 2015, 86: 085002

$10 \mathrm{Hu}$ B, Pang C K, Wan J, et al. Earliest switch-on of dual-stage actuation in hard disk drives. Microsyst Technol, 2016, 22: 1267-1273

11 Zheng J, Salton A, Fu M. Design and control of a rotary dual-stage actuator positioning system. Mechatronics, 2011, 21: 1003-1012

12 Zheng J, Tay F S, Lu R, et al. Robust sliding mode control design for a dual-stage actuator system. In: Proceedings of 2014 International Conference on Modelling, Identification \& Control. Melbourne: IEEE, 2014. 70-75

13 Zhang L, Long Z, Cai J, et al. Design of a linear macro-micro actuation stage considering vibration isolation. Adv Mech Eng, 2015, 7: 168781401558454

14 Zhang X, Wang C, Gao R X, et al. A novel hybrid error criterion-based active control method for on-line milling vibration suppression with piezoelectric actuators and sensors. Sensors, 2016, 16: 68

15 Dong W, Tang J, Eldeeb Y. Design of a linear-motion dual-stage actuation system for precision control. Smart Mater Struct, 2009, 18: 095035

16 Xu Q. Design and development of a flexure-based dual-stage nanopositioning system with minimum interference behavior. IEEE Trans Automat Sci Eng, 2012, 9: 554-563

17 Kim K, Choi Y M, Gweon D G, et al. A novel laser micro/nano-machining system for FPD process. J Mater Process Technol, 2008, 201: 497-501 
18 Choi Y M, Gweon D G. A high-precision dual-servo stage using halbach linear active magnetic bearings. IEEE/ASME Trans Mechatron, 2011, 16: $925-931$

19 Hiemstra D B, Parmar G, Awtar S. Performance tradeoffs posed by moving magnet actuators in flexure-based nanopositioning. IEEE/ASME Trans Mechatron, 2014, 19: 201-212

20 Elfizy A T, Bone G M, Elbestawi M A. Design and control of a dual-stage feed drive. Int J Mach Tools Manuf, 2005, 45: 153-165

21 Fang J W, Long Z L, Zhang L F, et al. Control analysis of point-to-point positioning based on macro-micro stage. In: Kim Y H, Yarlagadda P, eds. Advances in Mechatronics and Control Engineering, Applied Mechanics and Materials, Vols. 278-280. Stafa-Zurich: Trans Tech Publications Ltd., 2013. 1563-1569

22 Liu H, Lu B, Ding Y, et al. A motor-piezo actuator for nano-scale positioning based on dual servo loop and nonlinearity compensation. J Micromech Microeng, 2003, 13: 295-299

23 Koganezawa S, Uematsu Y, Yamada T, et al. Dual-stage actuator system for magnetic disk drives using a shear mode piezoelectric microactuator. IEEE Trans Magn, 1999, 35: 988-992

24 Chen X D, Zhang S Y, Bao X L, et al. Master and slave control of a dual-stage for precision positioning. In: 2008 3rd IEEE International Conference on Nano/Micro Engineered and Molecular Systems, Vols. 1-3. Sanya, China: IEEE, 2008. 583-587

25 Nagel W S, Clayton G M, Leang K K, et al. Master-slave control with hysteresis inversion for dual-stage nanopositioning systems. In: 2016 American Control Conference. New York: IEEE, 2016. 655-660

26 Guo G, Wu D, Chong T C. Modified dual-stage controller for dealing with secondary-stage actuator saturation. IEEE Trans Magn, 2003, 39: $3587-3592$

27 Liu K Z, Akasaka D. A partial parameterization of nonlinear output feedback controllers for saturated linear systems. Automatica, 2014, 50: 233239

28 Prasad S, Purwar S, Kishor N. H-infinity based non-linear sliding mode controller for frequency regulation in interconnected power systems with constant and time-varying delays. IET Gener Transm Dis, 2016, 10: 2771-2784

29 Schroeck S J, Messner W C, McNab R J. On compensator design for linear time-invariant dual-input single-output systems. IEEE/ASME Trans Mechatron, 2001, 6: 50-57

30 Seki K, Shinohara Y, Iwasaki M, et al. Force controller design based on PQ method for dual-stage actuators in polishing machines. In: 37th Annual Conference of the IEEE Industrial Electronics Society. Melbourne: IEEE, 2011. 3388-3393

31 Shinohara Y, Seki K, Iwasaki M, et al. Controller design for dual-stage actuator-driven load devices considering suppression of vibration due to input saturation. In: 2013 IEEE International Conference on Mechatronics. Vicenza: IEEE, 2013. 742-747

32 Shinohara Y, Seki K, Iwasaki M. Robust vibration suppression control for resonant frequency variations in dual-stage actuator-driven load devices. In: 2015 IEEE International Conference on Mechatronics. Nagoya: IEEE, 2015. 632-637

33 Yazaki Y, Fujimoto H, Sakata K, et al. Settling time shortening method using final state control for high-precision stage with decouplable structure of fine and coarse parts. In: 40th Annual Conference of the IEEE Industrial Electronics Society. New York: IEEE, 2014. 2859-2865

34 Li H Z, Le M D, Gong Z M, et al. Motion profile design to reduce residual vibration of high-speed positioning stages. IEEE/ASME Trans Mechatron, 2009, 14: 264-269

35 杨志军, 白有盾, 陈新, 等. 预应力梁等效刚度与固有频率的近似解析解. 中国科学: 物理学 力学 天文学, 2015, 45: 074601

36 Lee A Y, Choi Y. Smooth trajectory planning methods using physical limits. Proc Institut Mech Eng Part C-J Mech Eng Sci, 2015, 229: 21272143

37 Sencer B, Ishizaki K. Smooth polynomial interpolation for point-to-point trajectories with vibration avoidance. In: 41st Annual Conference of the IEEE Industrial Electronics Society. Yokohama: IEEE, 2015. 002070-002075

38 Pawlus W, Hansen M R, Choux M, et al. Mitigation of fatigue damage and vibration severity of electric drivetrains by systematic selection of motion profiles. IEEE/ASME Trans Mechatron, 2016, 21: 2870-2880

39 Tsay D M, Lin C F. Asymmetrical inputs for minimizing residual response. In: Proceedings of the 2005 IEEE International Conference on Mechatronics. Taipei: IEEE, 2005. 235-240

40 Rew K H, Ha C W, Kim K S. A practically efficient method for motion control based on asymmetric velocity profile. Int J Mach Tools Manuf, 2009, 49: 678-682

41 Rew K H, Kim K S. A closed-form solution to asymmetric motion profile allowing acceleration manipulation. IEEE Trans Ind Electron, 2010, 57: 
$2499-2506$

42 Li H. A jerk-constrained asymmetric motion profile for high-speed motion stages to reduce residual vibration. Int J Comput Appl Technol, 2016, 53: $149-156$

43 Yang Z, Bai Y, Chen X, et al. The residual vibration impact on the multiple target positioning time for array operation. In: Proceedings of the 2016 IEEE International Conference on Information and Automation. Ningbo, China: IEEE, 2016. 50-55

\title{
Design of rigid motion and elastic deformation parallel driven based macro-micro compounded positioning stage
}

\author{
BAI YouDun, CHEN Xin \& YANG ZhiJun
}

Guangdong Provincial Key Laboratory of Micro-Nano Manufacturing Technology and Equipment, State Key Laboratory of Precision Electronic Manufacturing Technology and Equipment, Guangdong University of Technology, Guangzhou 510006, China

High-speed precision positioning is highly demanded in electronic manufacturing, and the mechanical friction is the key factor affecting positioning accuracy. Currently, sub-micron positioning can only be achieved by using high-cost frictionless guiding system. A new parallel driven macro-micro compounded positioning stage design, which eliminates the saturation problem of micro actuator, is proposed by combining the long-stroke direct-driven stage and frictionless guiding mechanism based on flexure hinge. Two sets of driving and measuring systems are included in the macro-micro motion stage in order to control the macro and micro stages connected by flexure hinge separately. The high-speed and long-stroke movement is achieved by the rigid mechanical connection system of macro stage, and the flexible connection system of micro stage based on flexure hinge is used to quickly compensate the positioning error of macro stage. The above rigid-flexible motion scheme enables the long-stroke, high-speed and high-precision positioning. A new motion profile planning method based on the dynamic response optimization is also proposed to reduce the residual vibration of motion stage. Due to the faster response and lower tracking error performance of micro stage compared to the macro one, the macro/micro driving force is superimposed to realize the fast motion during the high-acceleration motion period and the micro-actuator can generate the inverse force to quickly compensate the motion error of macro stage during the constant-speed and low-speed positioning motion periods. Experimental results verified that the proposed macro-micro compounded stage design can effectively reduce the complexity of the control system through the innovation of the mechanism and realize high-precision and rapid positioning. It provides a new way for the design of sub-micron high speed positioning stage for micro-photoelectron manufacturing equipment.

rigid motion and elastic deformation, parallel driven, macro-micro compounded, precision positioning

doi: $10.1360 /$ N092017-00397 